\title{
Letter to the Editor regarding, "MIR196A2 rs11614913 contributes to susceptibility to colorectal cancer in Iranian population: A multi-center case- control study and meta-analysis"
}

\author{
Rama Jayaraj, Chellan Kumarasamy, K.M. Gothandam, Siddhartha Baxi
}

Dear Editor,

We have read the article, "MIR196A2 rs11614913 contributes to susceptibility to colorectal cancer in Iranian population: A multi-center case-control study and meta-analysis", published in the August 2018 edition of the journal, with great interest (Haerian et al., 2018). However, we would like to suggest a few improvements to observed oversights in the article, which hamper the utility of the study.

The authors primarily seek to establish the link between increased susceptibility to CRC and of MIR196A2 rs11614913, however they give no mention regarding the increased susceptibility of rs11614913 to NSCLC and Gastric cancer, which it is found to be highly susceptible to in previous studies (Yuan et al., 2013; Poltronieri-Oliveira et al., 2017). Furthermore, considering that the study attempts to highlight and evaluate the association between rs11614913 and CRC in a control group of Iranians, only 2 of the 14 studies included in the main meta-analysis were conducted in Iran, this leads to unreliable pooled effect estimates. The authors do include meta-analysis based individual countries as subgroups, and present pooled effect size estimates exclusive to Iranians, however, the small number of studies results in a low power of analysis, and subsequent uncertainty.

Also the study does not clearly demonstrate the effect of ethnicity on the distribution of the rs11614913 polymorphism in CRC, a genotype frequency analysis approach of rs11614913 polymorphism could be considered. The subgroup analysis was also found to be rudimentary with the only subgroups being based on ethnicity (Caucasian and Asian). Subgroup analysis could also seek to explore the high and low expression of the rs11614913 polymorphism.

These minor additions could serve to significantly improve the article and allow for others pursuing this line of research to use this study as a guidepost to future research regarding this topic. 


\section{References}

Haerian et al., 2018. M.S. Haerian, B.S. Haerian, S. Molanaei, F. Kosari, S. Sabeti, F. Bidarizerehpoosh, E. Abdolali. MIR196A2 rs11614913 contributes to susceptibility to colorectal cancer in Iranian population: a multi-center case-control study and meta-analysis Gene, 669 (2018), pp. 82-90 Poltronieri-Oliveira et al., 2017. A.B. Poltronieri-Oliveira, F.F. Madeira, D.B.S.M. Nunes, G.H. Rodrigues, B.C. Lopes, F.S. Manoel-Caetano, J.M. Biselli, A.E. Silva, Polymorphisms of miR-196a2 (rs11614913) and miR-605 (rs2043556) confer susceptibility to gastric cancer, Gene Rep., 7 (2017), pp. 154-163

Yuan et al., 2013, Z. Yuan, X. Zeng, D. Yang, W. Wang, Z. Liu, Effects of common polymorphism rs11614913 in Hsa-miR-196a2 on lung cancer risk, PLoS One, 8 (2013), Article e61047 